\title{
A Free - Floating Ball Valve Thrombus in the Left Atrium: Diagnosed Clinically and by Trans Thoracic Two - Dimensional Echocardiography.
}

\author{
Dr. J.Rajendra Kumar ${ }^{1}$, Dr. Mamta B. Kumbhare ${ }^{2}$, Dr. A. Akhila ${ }^{3}$, \\ Dr.Ch. Rachana ${ }^{4}$, Lt. Col. (Dr.) Sumanta Mandal ${ }^{5}$ \\ $\left({ }^{1}\right.$ Associate Professor, ${ }^{3}$ and ${ }^{4}$ PG students, Department of Medicine CAIMS, Bommakal, Karimnagar (A.P.) \\ India - 505 001) \\ ${ }^{(2}$ Senior Specialist Anesthesia, Dhanvanthari Hospital ,NTPC, RSTPS, Jyothi Nagar District Karimnagar (AP) \\ India-505 215 ) \\ $\left({ }^{5}\right.$ Physician, Military Hospital Shillong, 793001)
}

\begin{abstract}
A Free-floating ball valve thrombus in the left atrium is a rare clinical entity and has been reported infrequently in Indian literature despite the high incidence of Rheumatic mitral valve stenosis. Early diagnosis and prompt surgical intervention are mandatory otherwise it may lead to catastrophic consequence. We are presenting our experience with one such case which was diagnosed clinically and by Trans- Thoracic two dimensional echocardiography. This case report describe the clinical features and Trans- Thoracic two dimensional echocardiography(TTE) findings of a free - floating ball valve thrombus in the left atrium secondary to severe rheumatic mitral valve stenosis that was discovered incidentally in our symptomatic patient.
\end{abstract}

Key Words: Left atrium ball valve thrombus, Rheumatic heart diseases, Mitral valve stenosis.

Accepted Date: 10 July 2013

\section{Introduction}

Ball valve thrombus in the left atrium (LA) is a spherical clot which is freely mobile and intermittently occludes the mitral valve orifice. The formation of a free - floating thrombus within left atrium is a rare clinical entity. It usually occurs an in enlarge left atrium associated with a diseased mitral valve or after mitral valve replacement. Because of excess of their diameter in comparison to the mitral valve orifice, they are imprisoned in the LA. It is a rare condition with propensity for thromboembolism and therefore portends a sinister prognosis. An emergency non invasive cardiac investigation like echocardiography plays a major role in diagnosis of free-floating ball valve thrombus.

\section{Case Report}

A 50-year-old female admitted with medical history of palpitation and grade III dyspnea since 25 days and intermittently she becomes more dyspneic in sitting position. She was diagnosed rheumatic mitral valve stenosis several years earlier but she was not taking regular treatment. There was no history of syncope, neurological deficit, blood stained sputum, chest pain, abdominal pain, hoarseness of voice, and fever. On examination she was alert and her pulse rate was 86/minute, irregularly irregular, small in amplitude and all peripheral pulses were palpable. Peripheral cyanosis was absent. Her blood pressure was $110 / 70 \mathrm{mmHg}$ in supine position and in right upper limb. She had elevated jugular venous pressure ( $6 \mathrm{~cm}$ above the sternal angle).

Liver was $2 \mathrm{~cm}$ enlarged. Bilateral ankle edema was present. Cardiovascular examination revealed a grade II parasternal impulse, variable first heart sound, loud P2, soft early opening snap and a grade III mid diastolic murmur (MDM) with pre-systolic accentuation in mitral area in left lateral position. Intensity of MDM was changing from supine to sitting position and intermittently MDM disappear in sitting position. A pan systolic murmur in tricuspid area and ejection systolic murmur in pulmonary area were present. Bilateral basal crackles were present. Her electrocardiogram shows, irregular rhythm, irregular 'R-R' interval, absent ' $\mathrm{P}$ ' waves and undulation of baseline. X-Ray chest PA view shows cardiomegaly, straightening of left heart border and dilated pulmonary arteries (Figure no 7). Her complete blood picture, erythrocyte sedimentation rate, Prothrombin time (P.T. 9.1 seconds), International normalize ratio (I.N.R. 1.9), blood urea, serum createnine, blood sugar and complete urine examination were within normal limits. Trans -Thoracic two dimensional echocardiography was performed in all views which revealed severe mitral valve stenosis, enlarged left atrium and a free -floating ball valve thrombus. Mitral valve area (MVA) $0.81 \mathrm{~cm} 2$, which was measured by 
planimetry in parasternal shot axis (PSAX) view at mitral valve level ( figure no 2). Mean pressure gradient was $15 \mathrm{mmHg}$ at a heart rate of 86 per minute, without any mitral valve regurgitation. Parasternal long axis( PLAX) view shows typical " hockey stick appearance" of anterior mitral valve (AML) during diastole. Both leaflets of mitral valve are thickened and minimally calcified (figure no 1). Subvalvular apparatus was thickened (Figure no 1). Posterior mitral valve leaflet (PML) non pliable and AML was pliable (Figure no 1).

Aortic, Tricuspid and pulmonary valves were normal (Figure no 1\&5). M- Mode echocardiography during diastole shows, marked thickening of mitral valve leaflets, severely reduced EF slope $(3.00 \mathrm{~mm} / \mathrm{sec}$.) and fate EF slope. Trans mitral valve continuous wave Doppler (CWD) echocardiography in apical four chamber revealed increased pressure half time (271 milliseconds). Ball valve thrombus was spherical in shape, small in size $(1.23 \mathrm{~cm} \mathrm{X} 1.37 \mathrm{~cm}$ and area $1.73 \mathrm{~cm} 2)$, well demarcated borders and which was free floating (Figure no 3, 4 \&5). Ball valve thrombus was attached to AML on LA side (Figure no 4). The thrombus was protruding through mitral valve orifice into the left ventricle in diastole (Figure no $3 \& 5$ ), partially occluding the stenotic mitral valve (Figure no3 \&5)), interrupting the left ventricular diastolic feeling and during systole the ball valve thrombus rebounds freely back into LA (Figure no 4). Color Doppler echocardiography shows decreased blood flow through the stenotic mitral valve. Spectral Continuous wave Doppler recording at tricuspid valve reveled mild to moderate tricuspid regurgitation (T.R.), with peak pressure gradient $38 \mathrm{~mm} \mathrm{Hg}$ and mean pressure gradient $25 \mathrm{mmHg}$ at peak velocity of 3.08 meter per second (Figure no.6). Her systolic pulmonary artery pressure (SPAP) was $48 \mathrm{mmHg}$. We advised Trans esophageal echocardiography, invasive cardiac procedure ( like coronary angiography ) and mitral valve surgery with removal of ball valve thrombus but she refused.

\section{Discussion}

Left atrial ball valve thrombus is an important pathology. It is most often associated with rheumatic mitral valve stenosis. However it has been reported without mitral stenosis also [7]. Majority of reports about this condition are from Japan [8, 9].It is surprising that there are very few reports of this entity from India where Rheumatic mitral valve disease is rampant. In a study of 490 patients in Pondichery [10] with rheumatic mitral valve stenosis 169 were found to have thrombus in the left atrial body or appendage but non with ball valve thrombus.

Mitral valve stenosis is associated with left atrial thrombosis in $17 \%$ of patients and the addition of atrial fibrillation double the risk of left thrombosis [1].Patients with both mitral valve stenosis and atrial fibrillation who develop a left atrial thrombus are at risk for developing shortness of breath, syncope and even cardiac arrest if the left atrial thrombus completely occludes the stenotic mitral valve producing obstructive shock [2]. Most intracardiac myxoma is located in the left atrium (LA) and rarely may be associated with mitral stenosis [3]. LA myxoma most often attached to the intra-atrial septum and arises from fossa ovalis. LA myxomas are typically nonhomogenous in texture with lucent center or area of calcification. In case of LA myxoma leaflets are pliable and normal in thickness. In our case LA thrombus was attached to the AML, homogenous and none calcified. In our case TTE does not showed any mass in fossa ovalis and LA appendage clot.

Wood who first applied the term ball valve thrombus to this entity in year 1814, describe autopsy finding in 15 year - old girl with rheumatic mitral valve stenosis and syncope [4]. Recently the routine use of two dimensional echocardiography, three dimensional echocardiography, magnetic resonance imaging, computed tomography permits ante mortem diagnosis and therapy should thus be directed so that autopsy will no longer be an important means of diagnosis $[5,6]$.

A ball valve thrombus is thought to be originating as a small mural thrombus usually on the interatrial septum but rarely in the left atrial appendage and on mitral valve leaflets. The thrombus gradually enlarges and form a projecting mass that remains attached to the atrial wall by a pedicle. As the bulbous end of thrombus enlarges the pedicle lengthens and thins until eventually the thrombus separates or fragments. Thereafter as the thrombus spins freely in the atrium, it acquires its characteristic smooth, polished appearance. During thrombus development and subsequently morphological changes, it is very likely that the patient may experience transient embolism or stroke. Furthermore the movements of the thrombus may cause syncope or sudden death, depending up on the degree of mitral valve obstruction. Our patient did not experience any of these events although TTE showed a free-floating left atrium thrombus protruding through mitral valve orifice and spinning freely on its axis. Our patient had past history of rheumatic heart disease on irregular treatment and at the time of hospitalization she was in atrial fibrillation and severe mitral valve stenosis, so all these risk factors lead to development of ball valve thrombus.

Left atrial ball valve thrombus should be suspected if the patient with severe mitral valve stenosis and atrial fibrillation have intermittent or changing murmurs, syncope, mid -diastolic or pre-systolic murmurs may increase in intensity as a ball valve thrombus encroaches on the mitral valve orifice, and a mid-diastolic murmur may disappear temporarily when the orifice become obstructed. Variations in symptoms have been associated with changes in the posture or position of the patient. Exacerbation of dyspnea can occur when the patient is in 
sitting position. Our patent demonstrated these features except syncope. Her two dimensional TTE reveled thickened both mitral valve leaflets and subvalvular apparatus. AML pliable and PML were nonpliable. PSAX view at mitral valve level in diastole shows typical "fish - mouth appearance" opening of mitral valve. These entire echocardiography findings indicate she had chronic rheumatic heart diseases (RHD). In chronic RHD mitral valve stenosis is more common. PLAX, PSAX and apical four chamber views shows severe rheumatic mitral valve stenosis, a small spherical free floating ball valve thrombus [1]. Tran's tricuspid valve CWD shows mild to moderate TR and moderate pulmonary artery hypertension (PAH). Systolic pulmonary artery pressure (SPAP) was calculated by simplified Bernoulli equation \{Right Ventricular Systolic Pressure or SPAP in absent of significant gradient across pulmonary valve or RV outflow tract obstruction $=\mathbf{4}(\mathrm{V}) \mathbf{2}+\mathrm{RAP},(\mathrm{V}$ is the TR jet velocity in meter per second and Right atrium pressure is estimated from IVC diameter and respiratory changes) \}.Severity of mitral valve stenosis can be decided by various non invasive methods (Table no 1, 2, 3 and 4). In our patient we calculated mitral valve area by planimetric and pressure half time (PHT) methods. In patient with atrial fibrillation PHT is best measured from a recording with an adequate diastolic filling period or averaging different cardiac cycle. On the basis of classical clinical features, past history of RHD and TTE findings she was diagnosed as a case of free floating ball valve thrombus, secondary to severe rheumatic mitral valve stenosis, mild to moderate TR, moderate PAH, absent infective endocartitis vegetation and normal left ventricular function. Our patient was treated with Digoxin, Diuretic, Salt restriction, Warfarin, Penicillin G Benzathin at a dose of 1.2 million unit every 21 day and prophylaxis for infective endocaditis and she was symptomatically improved with this treatment and at the time of discharge we again advise mitral valve surgery and removal of thrombus but she refused ,then we advised regular follow-up .

\section{Conclusion}

When clinical evidence lead to the suspicion of a free floating thrombus, an echocardiography should be performed immediately. Anticoagulation and thrombolytic therapies do not appear to have a role in the acute management of left atrial thrombus, although the importance of anticoagulation in prevention of recurrence is obvious. Surgical removal of the thrombus with simultaneous treatment of the underlying cause is the first choice treatment with good result in most cases. Nowadays with the increase uses of non invasive cardiac investigation like echocardiography this entity can be diagnosed very easily and also identify its complication. It also helps in future treatment plan and prevention of the serious complications.

\section{References}

[1]. Rost C, Daniel WG, Schmid M. Giant left atrial thrombus in moderate mitral stenosis. Eur J Echocadiography. 2009;10:358-359.

[2]. Fan JS, How CK, Chery CH, Chen JD. Impending cardiac arrest due to mitral - valve stenosis and left atrial " ball-valve thrombus". Resuscitation 2007; 73: 328-329.

[3]. Sim EK, Lim YT, Ng WL, Goh JJ, Reebye S. Co - existing left atrial thrombus and myxoma in mitral stenosis - a diagnostic challenge. Singapore Med J. 1999; 40:46-47.

[4]. Wrisley D, Giambartolomeri A, Brownlee W. Left atrial ball valve thrombus: review of clinical and echocardiographic manifestations with suggestions for management. Am Heart J 1991;122:1784-1790.

[5]. Zur-Binenboim C, Ammar R, Grenadier E, Veisler A, Freud M, Palant A. Detection of round floating left atrial thrombus simulating left atrial myxoma by two dimensional echocardiography. Am Heart J. 1985;110:492-493.

[6]. Gottdiener J, Temeck B, Patterson R, Fletcher R. Transient occlusion of mitral valve by a free floating atrial ball thrombus : identification by two- dimensional echocardiography. Am J cardiol 1984;53:1730-1732.

[7]. Oryoji A, Kawara T, Hara H, Aoyagi S, Kosuga K, Ohishi K. Left atrial free- floating ball thrombus in a patient without mitral valve disease. Nippon Kyobu Deka Gakki Zasshi . 1993;41:699-703.

[8]. Aoyagi S, Kosuga K, Hisatomi K, Shima H, Tanaka I, Shimadu S, Ohishi K. Four successfully operated case of ball valve thrombus in the left atrium. ( Article in Japanese) Kyobu Geka .1989; 42:97-102.

[9]. Misumi T, Kudo M, Ito T, Matsubra T, Kumamary H. Floating ball valve thrombus in left atrium with mitral stenosis. JPNJ thorac Cardiovase Surg. 2003;51:387-389.

[10]. Srimannarayana j, Verma RS, Sathessh S, Anil kumar R, Balchandar J. Prevelence of left atrial thrombus in rheumatic mitral stenosis with atrial fibrillation and its response to anticoagulation. A Transoesophageal echocardiography study. Indian Heart J 2003; $55: 358-361$ 


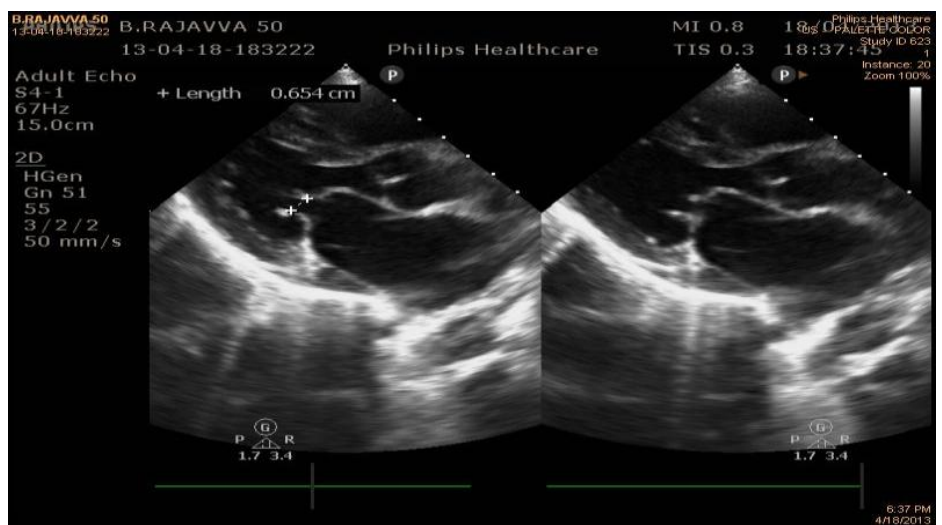

Figure No 1: PLAX view in diastole shows both leaflets of Mitral valve are thickened and typical "Hockey Stick" appearance .

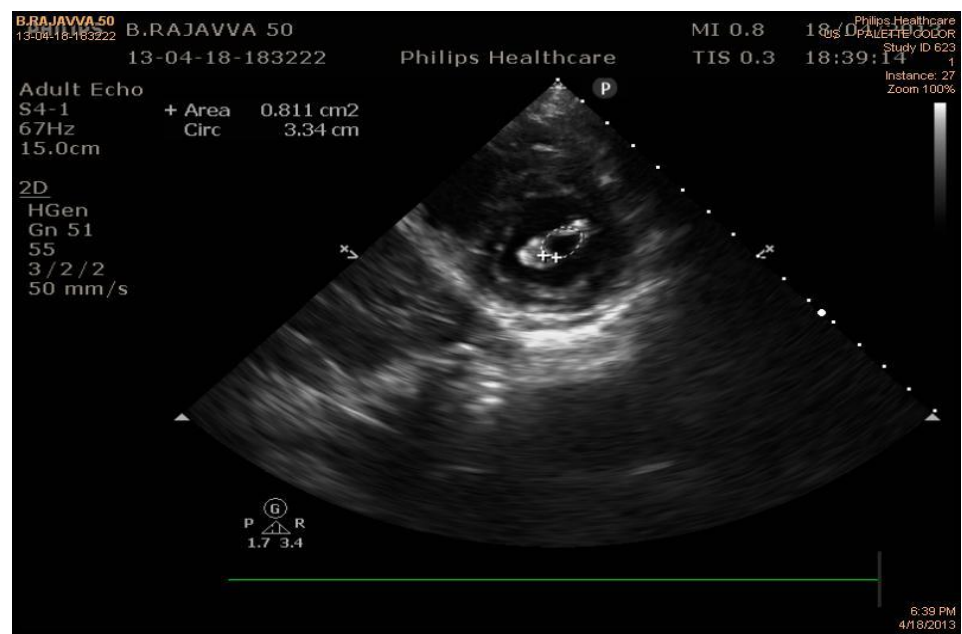

Figure No 2: PSAX view in diastole at Mitral valve orifice level shows "Typical Fish Mouth Opening Appearance" of severe Mitral valve stenosis in rheumatic heart disease.

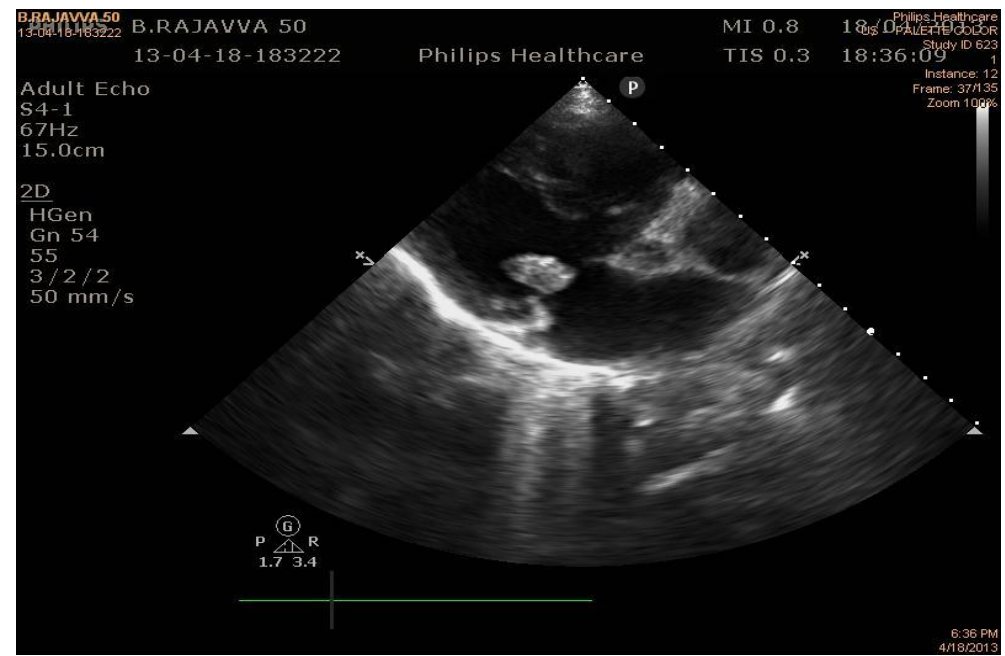

Figure No 3: PLAX view in diastole shows small size, spherical shape free-floating ball valve thrombus protruding through mitral valve orifice into the left ventricle and occluding the stenotic mitral valve and interrupting the left ventricular diastolic feeling. 


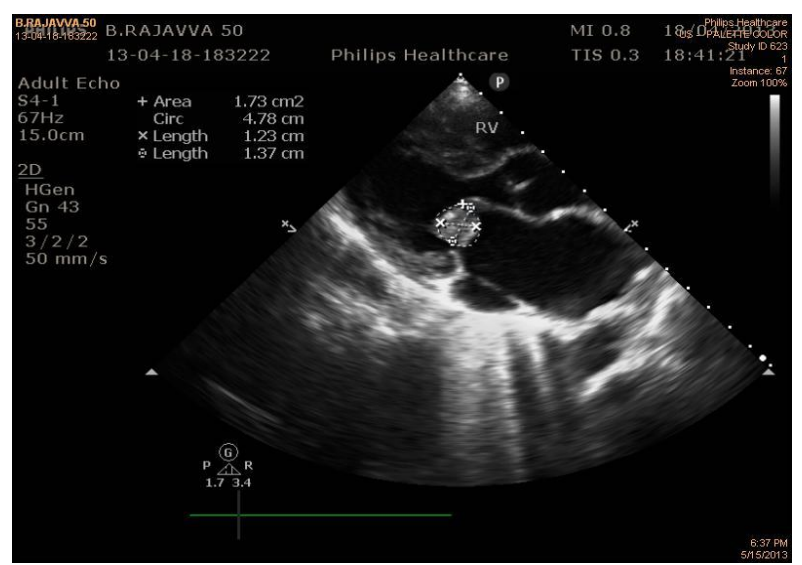

Figure No 4: PLAX view in systole shows small size, free-floating ball valve thrombus rebounds freely back into LA.

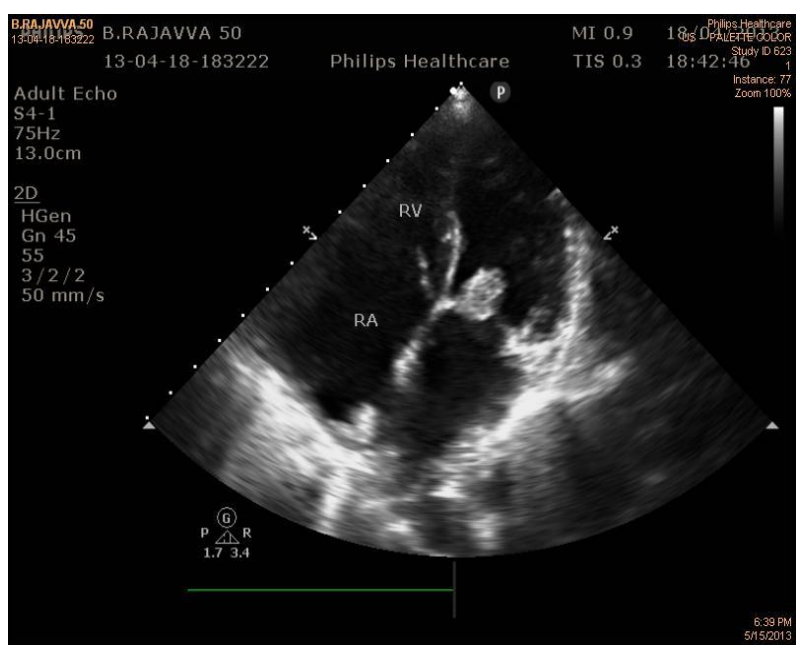

Figure No 5: Apical four chamber view in diastole shows a free-floating ball valve thrombus protruding through the mitral valve orifice into the left ventricle.( RV - right ventricle, RA - right atrium).

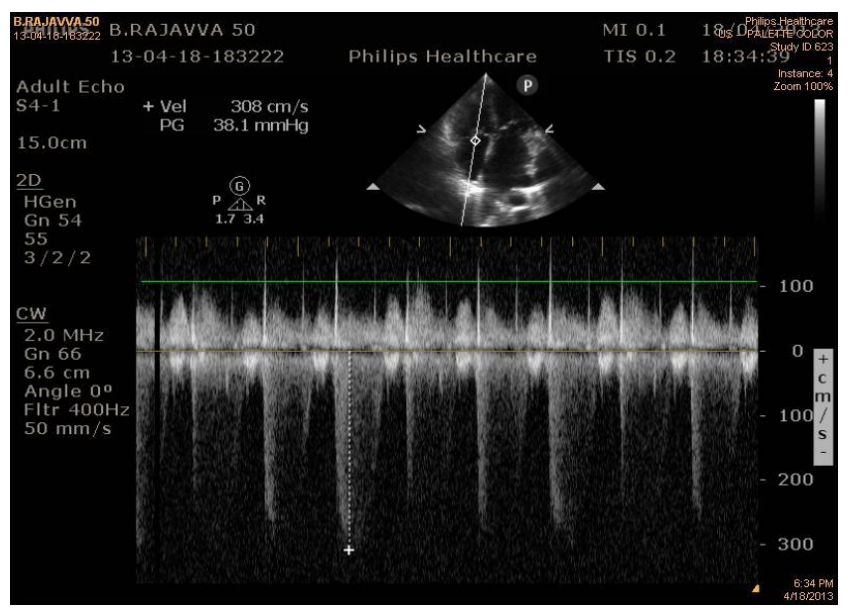

Figure No 6: Continuous wave Doppler recording at tricuspid valve level shows mild to moderate tricuspid valve regurgitation. 


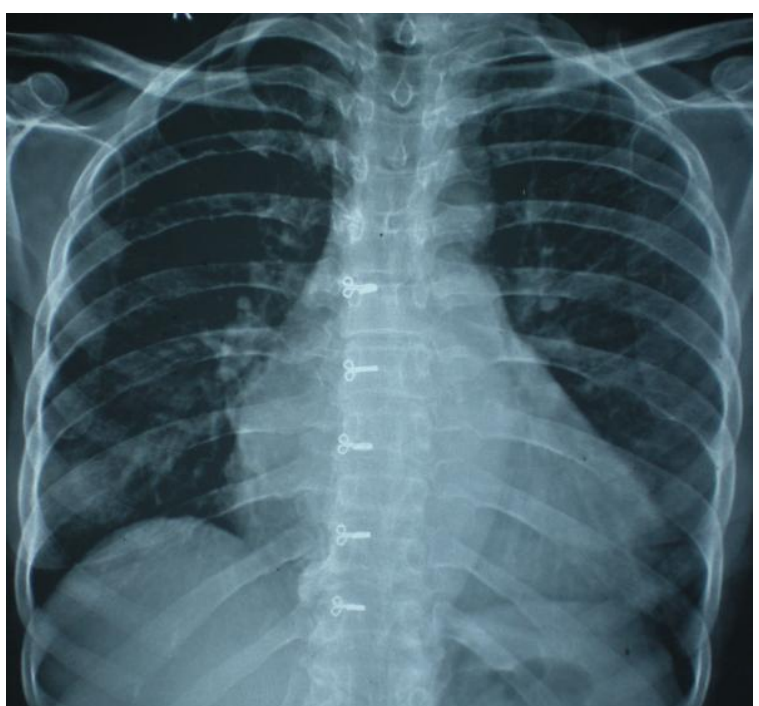

Figure No 7: X- Ray chest PA view shows cardiomegaly and striating of left border of heart.

Table No 1: Severity of Mitral Valve stenosis (M.S.) according to trans mitral valve gradient .

\begin{tabular}{|l|l|}
\hline Severity of M.S. & Trans valvular Gradient (mmHg) \\
\hline Normal Mitral Valve & $0 \mathrm{mmHg}$ \\
\hline Mild M.S. & $<5 \mathrm{mmHg}$ \\
\hline Moderate M.S. & $5-10 \mathrm{mmHg}$ \\
\hline Severe M.S. & $>15 \mathrm{mmHg}$ \\
\hline
\end{tabular}

Table No 2: Severity of Mitral Valve stenosis according to mitral valve area (MVA) and symptoms.

\begin{tabular}{|l|l|l|}
\hline MVA $(\mathbf{c m} 2)$ & Severity & Symptoms \\
\hline$>2.5$ & Minimal M.S. & No Symptoms \\
\hline 1.5 to 2.5 & Mild M.S. & Dyspnea on severe exertion \\
\hline 1.00 to 1.5 & Moderate M.S. & PND +/- Pulmonary edema \\
\hline$<1.00$ & Severe M.S. & Orthopnea ( NYHA Class IV) \\
\hline$<0.60$ & Critical M.S. & $\begin{array}{l}\text { Fatigue, Reactive PHT, } \\
\text { R.V. Failure }\end{array}$ \\
\hline
\end{tabular}

(PND - Paroxysmal nocturnal dyspnea, PHT - Pulmonary hypertension , R.V. - Right ventricle, Normal MVA 4-6cm2)

Table no 3: Severity of M.S. according to A2 - O.S. Interval (opening snap).

\begin{tabular}{|l|l|}
\hline Severity of M.S. & A2 - O.S. Interval \\
\hline Mild M.S. & 0.10 to 0.12 seconds \\
\hline Moderate to Severe M.S. & 0.05 to 0.07 seconds \\
\hline
\end{tabular}

(Normal A2 - O.S. Interval 30 to 150 milliseconds)

Table no 4: Severity of M.S. according to pressure half time (PHT).

\begin{tabular}{|l|l|l|}
\hline PHT(Milliseconds) & MVA $(\mathbf{c m} 2)$ & Severity of M.S. \\
\hline$>88$ & $>2.5$ & Minimal \\
\hline $88-145$ & $1.5-2.5$ & Mild \\
\hline $145-220$ & $1.00-1.5$ & Moderate \\
\hline 222 & $<1.00$ & Severe \\
\hline 370 & $<0.60$ & Critical \\
\hline
\end{tabular}

[ MVA can be calculated by use of Empirical formula (MVA $=220 /$ PHT). PHT is always proportionally related to deceleration time $(\mathrm{PHT}=\mathbf{0 . 2 9} \mathrm{X}$ DT $)$. ] 